\title{
Descensus ad Inferos: Taming (Easily) the Monsters in the Daunian Pictorial Tradition. The Exorcism of the Underworld and the Representation of Opposites Boundaries
}

\author{
Marisa Corrente
}

Funzionario Archeologo Soprintendenza Archeologia della Puglia

\author{
Doi:10.5901/ajis.2015.v4n1p457
}

\begin{abstract}
The city of Canusium (Canosa di Puglia) is one of the most important archaeological sites of southern Italy. The archaeological contexts of chamber tombs shed light on constructions of gender, personal identity, family relationships, and how the living interacted with the deceased. The major shift of daunian pictorial tradition occurs in the second centuries BCE with new decorative system and distinctive emphasis. In this paper, I analyze crucial components of the Daunian afterlife and the preferences of the iconographic evidence. In figurative Daunian imagery, the chamber tomb, liminal space for interaction between the living and the dead, clearly merits further investigation: it is not simply replicating some sort of house, but a tomb of the dead. The Daunian dimension of the Afterlife was marked by elements symbolic of passage identifiable in the doors of the tombs ant in that sort of interim space, antechamber of Hades(vestibulum). The burial chambers were a territory of unclear boundaries: the figured representation, the Hermes's psychopompic abilities, the high visibility of the women in procession, participants in the ritual, were intentionally positioned in the antechamber, in the cross-cultural association with death and the underworld. I argue that the terracotta statues of women in various gestures of praying or mourning, discovered arranged in small groups, were physical collaborators of the liminality. The daunian tomb is the word of the gorgon Medusa, apotropaic symbol: the snakey head of medusa in the representations on the vases with polychrome and plastic decoration has a crosscultural association with death and the underworld. The vase, showing snakes, chthonic elements, could be related to the apotropaic function and signify the ability to travel between worlds, the earth and the underworld.
\end{abstract}

Keywords: Funerary practices, journey to Hades, death and burial iconography and iconology, funerary customs, necropolis, tomb chamber, door, painted friezes, Hermes, deductio ad inferos, funerary cortege, iconographic program, local elite, social identity of the deceased, the Underworld, Cerberus, gorgon Medusa, nymphs, plural feminine, terracotta statues of women, vases with polychrome and plastic decoration, snakes

\section{II contesto}

L'archeologia funeraria del territorio daunio è ben nota per un sistema codificato di simboli ed atti, riflessi negli apparati strutturali e nella sequenza di oggetti pertinenti ai corredi, distintivi del genere e del ruolo dei defunti. La consapevolezza di un sistema identitario sta nel sistema di concatenazione e selezione, nella struttura costitutiva dell'insieme e nella successione materica di un linguaggio associativo, mezzo di espressione dei comportamenti sociali.

Gli esiti più significativi di questo processo culturale hanno avuto manifestazioni formali, come espressione di produzioni artigianali ben rappresentative dell'alto livello sociale di committenze di prestigio.

Si pensi come successione paradigmatica alla ricca sequenza di tombe gentilizie scavate nell'Ottocento e al processo di dispersione dei reperti, in gran parte vascolari, che ha disegnato una mappa museale a grandi maglie, legando complessi funerari di pregio ai più importanti musei del mondo. Dalla massa indistinta di informazioni, rappresentate dall'insieme di tombe e corredi della ricerca del passato ${ }^{1}$, si è passati nel panorama della ricerca odierna all'insieme di caratteristiche funzionali di un sistema funerario che distingue strutture ed aree di referenza, accentua il significato dei realia e dei dispositivi semantici alla base delle scelte comunicative.

Si tratta di una realtà complessa in cui è evidente lo sforzo di decodificare le forme dell'espressione e le forme del contenuto, definendo i rapporti tra significanti e significato.

In particolare, oggi è forte l'esigenza di riflettere sulla consistenza dell'ordine associativo dei contesti archeologici e di studiare l'insieme di convenzioni sociali che hanno prodotto un sistema di segni.

\footnotetext{
${ }^{1}$ Si veda, ad esempio, l'interesse di Johann Jakob Bachofen per le iconografie dei vasi policromi degli ipogei canosini
} rinvenuti nel fondo Lagrasta, oggetto di interpretazione filosofico-simbolica: Bachofen 2003. 
Occorre osservare che se l'interpretazione dei dati investe un campo come quello dell'archeologia funeraria in cui molteplici sono i fattori di mutabilità e di contaminazione, appare inevitabilmente condizionante l'approccio metodologico concettuale di partenza che può esercitare in maniera arbitraria cambiamenti di senso.

Questa necessaria premessa si impone e l'analisi qui operata acquista significato solo in relazione al processo di osservazione che ha evidenziato identità, contenuti ideologici e qualità differenziali in un sistema di associazioni specifico.

II valore documentale dell'archeologica funeraria della metropoli daunia di Canusium, centro primario dell'organizzazione insediativa della Daunia meridionale², riassume le osservazioni che seguiranno.

Ci è apparso, infatti, che la produzione segnica della realtà archeologica funeraria canosina costituisca come riconoscibilità, contenuto del campione e congruenza dello stesso una buona possibilità di analisi delle componenti degli atti di comunicazione che sono stati tradotti in oggetti e rituali. Si tratta di un dossier specifico, frutto di un processo culturale definito dagli assetti politico-sociali successivi alla "romanizzazione" del territorio.

L'ambito politico-sociale è quello di una società che rivendica la sua identità e il mantenimento dell'ordine, in una rete di partecipazione al nuovo ordine imposto dalla romanizzazione, necessariamente filtrato dalle complesse dinamiche di assestamento.

I dati attuali consentono di inquadrare le strutture in esame e le figurazioni funerarie nella fase postannibalica, nell'ambito del II secolo a. C. , in un secolo di straordinaria vitalità per la società daunia nel suo complesso3 3 .

\section{Gli ipogei come antri del rituale}

La vivacità delle manifestazioni funerarie nel mondo culturale daunio è strettamente condizionata dalla specificità spaziale e dai rituali svolti negli ambienti ipogei gentilizi. La creazione di un'architettura ipogea rende possibile la percezione di spazi strutturati e di percorsi sotterranei, con una netta distinzione tra paesaggio urbano e topografia funeraria ${ }^{4}$. Lo spazio ipogeo, in quanto spazio condiviso dai membri di un gruppo familiare, garantiva la comunicazione e costituiva un'unità dinamica di interazione, fondamento relazionale tra la comunità dei vivi e quella dei morti.

L'organizzazione spaziale può risultare complessa in rapporto all'articolazione interna delle tombe ${ }^{5}$, con divisione di camere, selezione di tipologie decorative, accentuazione del decoro dello spazio strutturato come riflesso degli stili di vita della comunità.

Chiaro e inequivocabile è il paradigma comunicativo a livello di distinzione planimetrica ed architettonica di una tomba a camera gentilizia(fig.1-2). Quello che si mette in scena è un simulacro del mondo sociale e un processo segnico di differenziazione delle immagini culturali più vitali.

Una serie di elementi allusivi, simbolici ed evocativi emergono in questa dimensione ostensiva influenzata da azioni e tracce: ogni comportamento ha valore di messaggio e nell'azione sociale del lutto l'etica della comunicazione ha sistemi codificati e mezzi di espressione.

Nel gruppo sociale di riferimento, lo spazio funerario è spazio strategico della comunicazione in cui si incrociano gli aspetti contraddittori legati all'evento importante della morte, alla lacerazione che si crea all'interno del corpo sociale e al lutto elaborato come desiderio di conservazione della società nel tempo.

Non secondaria e profondamente correlata all'ambiente ipogeo è la concezione di spazio scenico come rappresentazione di ciò che c'è oltre le soglie della visibilità.

Ora, occorre comprendere quali risorse, come categorie culturali, vengono utilizzate affinché la comunicazione verso il gruppo sociale abbia una costruzione di senso.

L'idea di partenza è la costruzione di uno spazio di rappresentazione del reale ${ }^{6}$. L'illusione di realtà è data dall'essere la tomba uno spazio chiuso da una porta, dall'imitazione delle travi lignee dei soffitti, dalle modanature architettoniche, dalla divisione degli ambienti. II livello di interfacciamento tra questo spazio che simula la realtà e il

\footnotetext{
2 L'organizzazione generale territoriale, basata su villaggi consorziali, fondati sulle unità insediative e sull'unità di stirpe, presuppone il controllo di terra agraria e l'esistenza di fondi di appartenenza a case gentilizie: Volpe 1990; Cassano 1992.

${ }^{3}$ Corrente 2012.

${ }^{4}$ Lippolis 1987: 39-154.

${ }^{5}$ Le tombe ipogee possono essere strutture a semplice vano con dromos di accesso o tombe a camera influenzate dall'adesione alle formule architettoniche elleniche. I tratti distintivi del processo di definizione del cosiddetto ipogeismo in terra daunia emergono in maniera significativa alla fine del medio bronzo ed hanno una formalizzazione continuativa tra l'età classica e il tardoellenismo ${ }^{6}$ Corrente 2003; Mazzei 1995.
} 
mondo dei vivi è nello spazio di passaggio, attraverso la porta, che separa due mondi, divide i soggetti vivi da quelli morti, ma nello stesso tempo dissolve la distinzione tra interno ed esterno.

Invero, lo schema dell'interfaccia è complesso. Vi è un sistema di regole, una grammatica generativa di come l'asse di comunicazione deve avvenire, una serie di stati attraverso cui passare.

Sono le strutture narrative, essenzialmente pittoriche, che aiutano nella comprensione del viaggio oltretombale. Si tratta di una tradizione iconografica ben definita, segno di una consapevolezza della propria cultura e di un radicamento di immagini che evocano il paesaggio e la topografia infera, suggerendo la rappresentazione dell'Ade.

Elaborazioni pittoriche significative, caratterizzate da omogeneità formale e decorativa, sono i fregi dipinti sui prospetti esterni delle tombe, espressione di una vocazione artigianale radicata nel territorio. Lo spazio del vestibolo è l'ideale scenario per il processo di nobilitazione dei defunti, in una logica di esaltazione della fama e della virtus pubblica degli stessi. La voluta enfatizzazione dei motivi guida si affida al richiamo di figure infernali, come Cerbero, e di divinità che storicizzano l'evento della morte, come Hermes psicopompo. Una preminenza visuale ha il defunto prossimo al suo cavallo. I fregi pittorici dell'ipogeo del Cerbero(fig.3) e dello Scocchera B7 (fig.4) sono profondante intrecciati per le analogie di schema iconografico, per la dialettica narrativa della solenne processione e per la condivisione delle componenti "istituzionali" del corteo. L'ordito pittorico che caratterizza il fregio dipinto sopra l'ingresso dell'ipogeo Sant'Aloia, notevolmente lacunoso, ha meno visibilità narrativa ma non si esclude che dovesse rispondere alle stesse coordinate di senso e di significato (fig.5). In ogni caso, le pitture, nella loro caratterizzazione simbolico-rituale, hanno una posizione centrale nella topografia funeraria, inserendosi in un punto di passaggio, lo spazio esterno del vestibolo, laddove l'osservazione è privilegiata ed è esplicita la volontà di veicolare messaggi a chi tra i vivi è rimasto custode delle case e dei beni dei defunti.

Di fatto, le scene pittoriche hanno la funzione di traghettare i defunti e nello stesso tempo rispecchiano l'unità sociale del gruppo familiare e del gruppo civico, non dissolta dall'evento della morte. Il contesto dipinto immette in una prospettiva infinita, in un orizzonte grandioso in cui l'oltretomba è vivo e ha suggerimenti inventivi e formali. Vi è una vis espressiva in questo allontanamento dall'umano, una lettura esplicativa di come sono costruiti l'architettura e lo spazio dell'oltretomba.

Singolarmente indicativo è l'accostamento della figura di Cerbero, il mostro trifauce ${ }^{8}$ a Hermes pychopompòs, ma il tumulto e l'orrore sono mitigati, in un movimento narrativo che vede il defunto come cavaliere che incede davanti al suo cavallo e un corteo simbolicamente rappresentato da un palafreniere (fig.6). Nell'esercizio di una tecnica pittorica che costruisce il racconto per segmenti accostabili secondo schemi variabili, l'animazione del tutto è apparente. Tutto è obscaenus, fuori scena, l'efficacia comunicativa è nella rivelazione ostentata. Viene messo in scena l'orrido, il pauroso, il mostruoso con Cerbero ringhiante ma anche l'immaginario eroico improntato ai valori aristocratici. Si esalta lo status di $e r o e^{9}$ del defunto, che ha raggiunto il traguardo della piena giovinezza, requisito necessario per la sua partecipazione alla guerra e culmine della vitalità, ma anche il legame genealogico dei viventi con il defunto, quella catena genealogica destinata a non interrompersi, nella cornice mitologico-ideale che segna il passaggio da un livello all'altro di vita ${ }^{10}$.

I personaggi delle scene pittoriche, quindi, sono eroi prototipici e le figurazioni, proprio in quanto paradigmatiche, mettono in scena il destino di morte degli eroi. II percorso ultraterreno verso il regno dei beati, in un orizzonte permeato da credenze misteriche legate all'orfismo e al dionisismo, sembra essere mediato dal tema escatologico del premio che consente agli eletti di stare con gli dei ${ }^{11}$.

Ora, accanto alla scene organizzate e sufficientemente drammatizzate dall'emergere del mythos e in complementarietà funzionale con il senso politico-mitologico dei cittadini-eroi, vi è un gruppo aggiuntivo a cui sembra essere affidato il compianto. Invero, due figure parrebbero "aggregate"e sottratte alla geografia funeraria e mitica del racconto pittorico.

Si tratta di un fuori scena di personaggi femminili stanti, spettatrici dirette dell'evento, presenti sia nella deductio ad inferos dell'ipogeo del Cerbero sia nella scena dell'ipogeo Sant'Aloia. La coppia di donne non è certamente un elemento

\footnotetext{
7 Sugli ipogei con pitture funerarie: Corrente, De Leo, Di Stefano, Nervo 2011, con bibliografia: 309-328.

${ }^{8}$ La tradizione non è concorde sul numero di teste che passano dalle cento alle cinquanta fino al numero di tre dell'iconografia più tradizionale. In Omero c'è già un terribile cane di Ade (Iliade, VII, 368; Odissea, XI, 623) che non viene nominato. Cerbero come aspetto e funzioni è descritto da Esiodo nella Teogonia (Theogonia, 310-312).

${ }^{9}$ Brelich1958.

${ }^{10}$ L'oblio serve ai morti per ritornare in vita: Plat. Rsp. 621 a. Hermes, il dio psicopompo per eccellenza, darà l'immortalità al proprio figlio concedendogli di conservare la memoria anche dopo aver attraversato l'Acheronte: Apoll. Rhod. Arg. I, 643 s.

11 Plat., Phaed. $69 \mathrm{c}$.
} 
secondario nella dinamica spaziale pittorica e, pur non entrando in gioco con un ruolo attivo nella scena, riveste una funzione fondamentale. Vi è nell'impianto iconografico una consapevole cesura che dà volto al femminile, certamente uno sdoppiamento della femminilità ma anche un femminile pacificato. Questa ricerca di un mondo "altro" ha consolidate conferme iconografiche come documenta il ricco repertorio di immagini plurime di figure efebiche o femminili, dai vari atteggiamenti, poste su piani distinti ai lati dei naiskoi ${ }^{12}$ nella produzione ceramografica a figure rosse.

La peculiarità dei cosiddetti "offerenti" sui vasi italioti non è scontata, tanto da non rendere univoca nella storia degli studi l'interpretazione di paides e parthenoi complementari e dialetticamente opposti ai soggetti principali entro lo spazio sacrale del naiskos. La riproduzione dei naiskoi nella ceramica italiota esalta, infatti, la centralità di uno spazio architettonico, espressione metaforica, come tomba-santuario, dell'elevazione del defunto e della glorificazione gentilizia. Per gli offerenti all'esterno dello spazio monumentalizzato, si è proposta l'identificazione con figure di beati, in gran parte protagonisti di funzioni e culti domestici, custodi delle case e in stretto rapporto con i defunti eroizzati ${ }^{13}$. L'assenza di tensioni e stati conflittuali è ben rimarcata dalla pacata gestualità e dall'intensità coreografica dei repertori figurativi che dilatano sulle superfici delle ceramiche della produzione tardoapula il numero dei partecipanti a rituali in cui sono forti le componenti sacrificali e l'esperienza erotica e dionisiaca.

Questa particolare liturgia funeraria, in cui si mescolano giovani di entrambi i sessi, resta, comunque, problematica in uno spazio pittorico che ripropone essenzialmente oggetti della sfera domestica e della scenografia cittadina. Di fatto, i personaggi costituiscono presenze transiconografiche ed entità impersonali, apparentemente prive di pathos ed emozioni, tutte protagoniste della medesima storia che pone la questione del confine e della dialettica tra interno ed esterno.

In quest'ottica, significativa è la riduzione della comunità delle offerenti al duale della scene funerarie dei monumenti canosini, con una finitezza che sembrerebbe sottolineare l'articolazione parentale dei legami sociali della famiglia. Ripercorrendo l'analisi esegetica sul ruolo svolto dalle due donne, pacate accompagnatrici del defunto, si richiama come l'interpretazione tradizionale evidenzi il forte legame con il defunto, con una soluzione pittorica che esplicita nell'immagine femminile, prossima alla figura eroica, la legittimazione delle prerogative funzionali al ruolo rigidamente muliebre, in un contesto di compianto funebre.

In ogni caso, non è da escludere che il codice culturale alla base della raffigurazione sia più complesso.

Come forme simboliche, le due donne lasciano emergere quel femminile "patetico" che garantisce l'attualità e divide culturalmente vivi e morti14. Qui occorre, comunque, far emergere la dimensione storico-sociale della distinzione operata, tra il luogo in cui vi è la costruzione mitologica-simbolica e il luogo di scambio e contatto in cui la presenza femminile è pensata al limite. II punto è che vi è una marcata differenziazione rispetto alla prospettiva narrativa dei fregi pittorici nella netta separazione delle due donne che ha forse una precisa motivazione culturale nella posizione delle figure sul lato sinistro e in basso. Si percepisce, nella posizione di questo spazio muliebre, un evidente stato di minorità che richiama lo sbilanciamento del femminile ${ }^{15}$ e la dicotomia dei principi opposti delle opposizioni polari dei Presocratici.

La donna, la cui natura femminile è fisiologicamente inferiore, è infatti una specie a parte e mantiene una netta separazione dagli uomini-maschi ${ }^{16}$. Nella tavola pitagorica dei contrari, che Aristotele riproduce nella Metafisica, $\ll<$ la femmina è posta nella stessa colonna dell'illimite, della sinistra, del brutto, del buio, contro la colonna nella quale, assieme al maschile, compaiono il limite, la destra, il buono, la luce >>17.

La collocazione delle figure, estrema, al limite, come secondarietà del femminile, sembrerebbe necessaria: la polarità intrinseca delle donne permette di dare fisicità a questo limite che indica la soglia tra la vita e la morte ${ }^{18}$.

Sulla base delle osservazioni proposte, il presupposto del substrato culturale è la natura concettuale della

\footnotetext{
12 Lo schema iconografico del naiskos viene introdotto nella ceramica italiota intorno alla metà del IV secolo a.C.: Pontrandolfo et al.1988,181-202; Esposito, Pedrina 2003:182: «avec l'iconographie du naiskos présence et figuration du défunt sont perçues de façon complémentaire selon un schéma que l'on pourrait qualifier de re-présentation ».Per la tipologia architettonica del naiskos, monumento su alto podio destinato alle classi medio ed alte ateniesi, adottato dall'aristocrazia tarantina tra IV e II secolo a. C. cfr. Lippolis 2007:22-40.

13 Schmidt 1996; Massa-Pairault 2009: 196.

14 Chirassi Colombo 1995: 47-82.

${ }^{15}$ La differenza biologica è assoluta in Esiodo. Secondo il racconto degli Erga, Hermes insediò in Pandora un "kyneos ... noos", una mente canina e una indole ingannevole ( Hes. Op. 67).

16 Héritier-Augé 1993: 128-29.

17 Aristot. Metaph. a 986 a; Lloyd 1993:49-85; Franco 2008,218. Il motivo del collegamento tra lato sinistro e genere femminile è culturalmente diffuso. Cfr. l'analisi sulla parte destra "buona" nelle lamelle orfiche in Battezzato 2005.

${ }^{18}$ Si veda in Héritier-Augé 1993 l'analisi della donna come "porta dimensionale": 231.
} 
distinzione resa manifesta dall'enfasi posta nelle proporzioni e dall'alterità estrema. Proprio sulla base di questo presupposto, risulta significativo, nell'analisi dei legami relazionali, porre l'accento sulla possibilità di una diversa cifra interpretativa della presenza femminile che in maniera fortemente allusiva potrebbe declinare una sfumatura estranea alla dimensione coniugale, richiamando la particolare essenza delle ninfe ${ }^{19} 0$ delle Sirene, figure a metà tra l'umano e il divino, esseri dalla doppia natura, simili a spiriti elementari20.

Le ninfe figure della marginalità incarnano la linea d'ombra tra umano e non umano e appaiono propedeutiche ed ausiliarie al viaggio oltremondano. Sono creature che per la intrinseca bellezza possono costituire oggetto della passione amorosa, simbolo della pulsione amorosa e del rapimento erotico ed iniziatico degli efebi. Le ninfe con la loro polarità intrinseca permettono di dare fisicità al limite che indica la soglia tra la vita e la morte.

In questa prospettiva di lettura, la versatilità simbolica della femminilità plurale verrebbe ulteriormente rafforzata da una presenza di rilievo dell'archeologia funeraria canosina, componente esclusiva della storia di quel territorio. Un ruolo chiave nella formazione del corredo funerario delle tombe a camera ellenistiche viene, infatti, assunto dal nucleo fittile di figure femminili ammantate, che enfatizzano nelle proporzioni e nello schema iconografico, la peculiare e ricca espressione plastica dell'artigianato canosino.

La tradizione artigianale locale si era già felicemente combinata, nelle piccole terrecotte figurate, al linguaggio pittorico dei vasi policromi dipinti a tempera ${ }^{21}$. La produzione statuaria è stata ricondotta ad un'esperienza coroplastica circoscritta nel tempo, non più di due generazioni di artigiani che producono statue alte circa $90 \mathrm{~cm}$ per una committenza di altissimo livello22.

Rinvenute all'interno dell'ambiente-atrio, nodo centrale dell'organizzazione interna delle camere, costituiscono una piccola folla di donne, un coro in cui rientrano anche jeunes filles, figure segnate dalla accentuazione patetica dei volti e da una precisa gestualità. Nella codificazione del tipo, l'esaltazione di una mestizia accentuata dalle braccia portate in avanti 0 strette al petto ha comportato l'identificazione del gruppo come oranti o piangenti di Canosa, forme espressive del planctus funebre(fig.7-8). Nella concretezza della rappresentazione e nella finzione mimetica, le statue sembrano svolgere una funzione stabilizzante e conciliatrice. II numero delle statue è variabile, con un orientamento preferenziale per la doppia coppia, fino ad arrivare agli otto esemplari dell'ipogeo Scocchera B. II corredo scultoreo anima la geografia funeraria e definisce, nella preminenza visuale, la centralità di una processione nevralgica nella sintassi cultuale. L'esibizione di componenti spettacolari dal punto di vista della scenografia è invero in stretto rapporto strategico con lo spazio infero. Apparentemente esse si muovono verso i viventi, con un ruolo di trasformazione unificatrice dello spazio, in realtà il coro di donne si muove nella direzione simmetricamente opposta, oltre le soglie della visibilità. Non ci sembra trascurabile questo contesto femminile "allargato", laddove il femminile al plurale, in questa dimensione ctonia, è originarietà e ripetizione, forma e materia.

Perfettamente vestite con chitone e himation, moltiplicano all'interno della tomba l'esperienza singolare della coppia di donne dello spazio pittorico esterno, con pari compostezza nell'abbigliamento, ma con maggiore vitalità gestuale. Una distinzione importante ha riguardato la classificazione per classi di età. Come giovani spose sono state identificate quelle che hanno una tunica stretta da una cinta alta ${ }^{23}$ e chiome sciolte. Si tratta di creature dal notevole valore simbolico, un silenzioso corteo che presiede ai passaggi tra i mondi, tra l'aldiquà e la morte, tra l'adolescenza e l'età adulta, tra l'umano e il soprannaturale. II ruolo delle statue è indubbiamente simbolico nell'allusione alla verginità artemidica ed adolescenziale, propria delle fanciulle che non hanno assunto un ruolo definito all'interno del Kosmos

\footnotetext{
19 Larson 2001.

20 Mancini 2005; Breglia Pulci Doria 1987:65-98; Breglia Pulci Doria 1990: 63-78.

21 Van Wonterghem-Maes 1968.

22 Cfr. Biardot 1872: 364: "exprimant la douleur... Leur robe est rayée de bandes blanches et pourpre rose alternées. Elles ont été trouvées dans la chambre d'apparat no 1 du tombeau dit de Medella où elles étaient placées vers la tête de la défunte ॥; Oliver 1968; Van der Wielen 1999: 43-65; Ferruzza-Giacommara 1993: 71-82. II repertorio è stato oggetto di un'ampia ricognizione e di analisi puntuali in Jammet 2003: 255-292.

${ }^{23}$ Cosi nel mondo romano la giovane sposa indossava la tunica recta stretta con una cintura di lana legata in vita con un nodus detto Herculeus: Plin. Nat. Hist. 28, 63-64; Macrob. Sat. I, 19, 16.Tale nodo era detto anche nodus herculaneus (Sen. epist. 87, 38). Questo particolare nodo doveva essere sciolto la sera delle nozze in lecto dallo sposo, invocando luno Cinxia o Cincia. II nodo aveva con funzione apotropaica e propiziatoria, come augurio alla fecondità testimoniato anche dal puntuale riferimento delle fonti alla numerosa prole di Hercules. Cfr. Bettini 1998:114-120.
} 
sociale, contrapposta alla maturità della donna sposa e madre ${ }^{24}$. L'aspetto rituale e collettivo ci sembra di particolare interesse, come tematizzazione di una femminilità plurale, ma altrettanto evidente è la peculiarità ambientale, I'"altrove"oscuro ed umido della tomba sotterranea, un habitat in cui l'esclusività del gruppo coroplastico evoca la questione della composizione dei tiasi e delle competenze cultuali delle donne. Esse vivono in una dimensione segreta ed oscura, che richiama quell'antro delle Ninfe omerico dove le Ninfe Naiadi incessantemente tessevano stoffe di porpora su telai di pietra ${ }^{25}$.

Ci sembra, invero, che la cornice di ambientazione in cui si muove questo coro femminile ${ }^{26}$ sia consono alle Ninfe $^{27}$. Inoltre, viene amplificato, con la presenza di due distinte tipologie di statue, il tema dell'eros e del passaggio da uno status all'altro. All'aspetto seduttivo delle statue-ragazze si contrappone la convenzione coniugale e materna delle statue-spose ${ }^{28}$. Coerentemente con l'aspetto seduttivo delle Ninfe, figure liminari, al di fuori di ogni convenzione umana 0 divina, avviene il rapimento erotico e iniziatico degli efebi.

D'altra parte, l'ambiguità "inquietante" di questo repertorio coroplastico, apparentemente semplificato nell'esaltazione di un femminile che assolve la sua funzione in gruppo, può suggerire ulteriori spunti di riflessione. La presenza delle statue come cogenti implicazioni di un linguaggio cultuale risulta evocativa a vari livelli. Una riprova di un certo grado di complementarità del complesso fittile con l'aldilà pitagorico è nel numero delle statue. Particolarmente suggestiva, nel mito conclusivo della Repubblica di Platone, ispirato alla speculazione pitagorica, è l'intrinseca coerenza filosofica implicita nel numero di otto Sirene ${ }^{29}$ garanti dell'armonia del moto delle sfere celesti. Poste su un disco che ruotava attorno a un fuso poggiato sulle ginocchia di Ananke, ciascuna Sirena emetteva un suono, cosicché ne scaturiva un'unica armonia ${ }^{30}$. Le Sirene in Platone sono portatrici di armonia ma già in Omero sono una voce che canta e un sapere che viene cantato. II loro canto, foriero di pericoli, arresta il tempo e provoca l'oblio. La loro è una capacità, tutta al femminile, di dire la storia, di dare senso alle azioni attraverso un discorso cantato. Nello spazio funerario dell'ipogeo Scocchera B, le otto statue femminili potrebbero rievocare la stessa strategia comunicativa del racconto di Platone, con un'accezione consapevole al sapere e all'armonia della conoscenza. L'insieme figurativo, nell'accorgimento numerico non casuale, potrebbe suggerire la ricezione di dottrine orfiche e la partecipazione di gruppi femminili gentilizi a cerimoniali particolari, secondo una scenografia cittadina e regolamenti cultuali31 che a noi risultano problematici ma che dovevano saldarsi a divinità tutelari della città.

\footnotetext{
${ }^{24}$ Nella rete di allusione ad eventi e cerimonie della vita sociale, appare esplicita la volontà dei committenti di richiamare la partecipazione a rituali religiosi collettivi. II valore allusivo delle raffigurazioni plastiche potrebbe costituire il riflesso di rappresentazioni reali in cui le protagoniste erano donne al vertice dei gruppi aristocratici.

${ }^{25}$ L'antro delle Ninfe descritto in Omero (Od. XIII, 102-129), la grotta dinanzi alla quale i Feaci lasciano Ulisse addormentato, ha un notevole valore simbolico.

${ }^{26}$ Calame 1997.

27 Si veda la pluralità delle ninfe teocritee: Theoc. 13. 39-60.

${ }^{28}$ Andò 1996: 47-79.

${ }^{29}$ Propedeutiche ed ausiliarie al viaggio oltremondano (come ippocampi, sfingi, cavalli alati, uccelli,), portatrici della morte, le sirene si caratterizzano per abitare alle soglie dell'Ade ed essere protettrici della tomba: Breglia Pulci Doria, 1987,65-98; Breglia Pulci Doria 1990: 63-78.

30 Plat., Rep. X, 615c-617.

${ }^{31}$ Non si può non rilevare come emerga nella presenza di gesti e simboli propri della componente femminile il possibile riferimento a pratiche rituali e al carattere pubblico di cerimonie, in cui le donne potevano assumere il ruolo di mediatrici tra la città e le divinità. Si pensi alla presenza delle donne, come rovesciamento delle convenzioni sociali, nei complessi rituali ateniesi legati alla celebrazione di Dionysos. Associazioni importanti, come le quattordici gerarai preposte alla preparazione del rituale negli Anthesteria, acquistano un valore documentario, soprattutto per la presenza della figura della basilinna: Nilsson1916,201. La presenza di collegi femminili ha un rilievo forte anche in altri ambiti cultuali greci con il ruolo di responsabilità nella guida dei cerimoniali svolto dalle sedici donne Elee 0 dalle Tiadi delfiche che avevano l'incarico rituale di richiamare il dio dagli inferi. La distinzione leggibile nei fittili canosini tra fanciulle e spose potrebbe avere un'intrinseca specificità e rappresentare l'effettiva composizione di gruppi di donne nelle pratiche cultuali. Si veda, a tale proposito, in Diodoro Siculo (Biblioteca storica, IV, 3, 2-3) la dimensione di gruppo dei riti femminili dionisiaci con le "vergini» (parthenoi), preposte a portare il tirso, urlare l'evoé, e le «donne» (gynaikai) incaricate di fare sacrifici e di cantare inni per l'apparizione del dio. Nei bakcheia descritti da Diodoro, fondamentale è la differenza tra il «raggiungere l'invasamento» (synenthousiazo) per le vergini e il «baccheggiare» (bakcheuo), come apertura all'eccesso, per le donne già sposate.
} 


\section{Vasi funerari con serpenti}

Una straordinarietà unitarietà di ideazione pittorica unisce tre askòi32 (figg.9-11) su cui occorre riflettere per la peculiarità del soggetto e la rispondenza dello stesso al particolare clima ideologico e culturale della fase post-annibalica. La scelta figurativa merita particolare attenzione per la non trascurabile novità della manifestazione pittorica. La rappresentazione si inserisce in quella particolare categoria di vasi, afferenti alla classe ceramica policroma con decorazione plastica, in cui la presenza della maschera di Medusa, nelle fattezze rassicuranti del tipo bello, dà valore al contenitore, legato all'acqua, come 'vaso-volto'.

In questi vasi che si fanno interamente prósopon, viene dipinta sul collo una coppia di serpenti in posizione araldica in cui è evidente, nel colore bluastro, la putredine che corrompe il corpo. La rappresentatività simbolica dei vasi cerimoniali, con funzione esclusivamente funeraria, ha indubbi aspetti innovativi nella scelta della raffigurazione dei serpenti effettuata dal Pittore degli askòi con festoni, con esiti perfettamente convergenti con gli aspetti teatrali e simbolici della pittura funeraria.

Risulta fondamentale porre l'accento sulla capacità del pittore di rinnovare il repertorio figurativo. Aspetti morfologici e l'introduzione di specifici motivi ornamentali, quali i festoni con bende, consentono di riportare all'atelier della prima metà del II secolo a.C. una serie di vasi distinti da aspetti iconografici e formali unitari ${ }^{33}$.

Dal punto di vista della composizione d'insieme, plastica e pittorica, si avverte una fortissima assonanza formale tra la realizzazione plastica e l'esito pittorico, in un'evocazione dilatata e vigorosa dei serpenti attributi della Gorgone, realizzati dall'artigiano come appliques. II paradigma relazionale Gorgo-serpente è strumento essenziale per la decifrazione del tratto iconografico caratterizzante. La scelta del soggetto e l'immagine dei serpenti isolata all'interno del repertorio pittorico della ceramica policroma; la collocazione della coppia in un spazio inquadrato da rami34 dipinti di nero; la posizione araldica dei rettili; il colore delle carni, precisa allusione agli aspetti mortuali dei corpi, sono tutti elementi che evidenziano la cifra peculiare della composizione in cui risulta essenziale il legame con l'ambiente infernale.

Per apprezzare l'orizzonte simbolico del serpente nell'elaborazione culturale della grecità che ne sottolinea la presenza privilegiata, può essere utile ripercorrere brevemente il ruolo svolto dal serpente nel mondo mitico, suggerendo la chiave interpretativa di un'animalità non secondaria, punto di confluenza di numerosi apporti ideologici e struttura simbolica atemporale.

L'essere figlio della terra, il suo scomparire e riapparire periodico, la sua capacità rigenerativa dovuta al cambio della pelle portano ad associare il serpente al ciclo della morte e della rigenerazione ${ }^{35}$. Un elemento fondamentale, nella costruzione speculativa, è il rilievo ctonio dell'animale. Occultato sotto la superficie della terra, il serpente sembra un simbolo molto adatto a rappresentare la morte e la vita dopo la morte e come tale esso si addice alla cornice aulica degli eroi. Come Medusa che nasconde il volto, il suo compito è quello di spaventare e di muoversi nello spazio proprio dell'orrido ${ }^{36}$. II serpente compare nei culti di Demetra Thesmophoros in relazione alla crescita dei raccolti e della progenie umana ${ }^{37}$. Nei thesmophoria i serpenti sono a guardia «delle parti inaccessibili del santuario» ${ }^{38}$ e stanno intorno alle voragini (megara) dove vengono gettati i maialini a putrefare.

Nell'orizzonte categoriale del "mostruoso", i serpenti sono segno di una dimensione "altra" tanto da custodire i confini della terra ${ }^{39}$ ed avere dimensioni cosmiche, come Typhon, entità di particolare grandezza, figlio di Hera 0 di

\footnotetext{
${ }^{32}$ La serie è composta dall'askòs cat.A/1.1.6 dell'ipogeo dei serpenti piumati, dall' askòs al Museum fur Kunst und Gewerbe di Amburgo e da un terzo vaso del Museo Civico di Canosa: Corrente 2003:47.

${ }_{33}$ Si tratta di produzioni che traggono ispirazione in un repertorio decorativo mutuato dall'ambiente tarantino e diffuso a Canosa nell'ambito di un'esperienza artigianale ceramica di breve durata.

${ }^{34}$ Non si esclude l'affinità di questi motivi vegetali con rami di palma.

35 Samonà 1991.

36 Sancassano 1997: 355-390.

${ }^{37} \mathrm{Nel}$ rituale tesmoforico, nel terzo giorno di Kalligeneia, le donne manipolano figurine di pasta dura a imitazione di serpenti e organi sessuali: Detienne 1982: 139. Un modellino plumbeo di serpente è stato trovato nel temenos di Demetra di Evreokastro a Thasos, come rappresentazione di Zeus Ktesios. Zeus è onorato nel santuario assieme ad Atena, alle Ninfe ed Artemide ed ha l'epiteto patroos, per $i$ suoi rapporti con i culti familiari e civici. In quanto ktesios, egli è il daimon protettore del focolare e della camera delle provviste ed è raffigurato come un serpente: Rolley 1965:452-456.

${ }^{38}$ Scolio a Luciano, Dialogo delle Meretrici, 14-19.

${ }^{39}$ Esiodo, Teogonia, 332-335.
} 
Gea ${ }^{40}$.

La nozione di terra e il suo possesso esplicitano nell'immaginario mitologico greco lo stretto rapporto tra terrigenia e serpente. L'associazione serpente-eroe è ampiamente attestata in letteratura e il combattimento con un mostro ofiomorfo è premessa di fondazione di città, come Cadmo a Tebe ${ }^{41}$.

I due piani della natura e della società, dell'ordine cosmico e dell'ordine civile, vengono superati nella legittimazione del percorso che afferma un ordine nuovo: Erittonio, il secondo re di Atene, era un antico un serpente nato dalla terra ${ }^{42}$ e Cecrope il primo mitico re della città era immaginato con la parte inferiore del corpo a forma di serpente ${ }^{43}$. Il serpente-drago è simbolicamente ricorrente nella storia degli oppositori tebani di Dioniso. Dal drago ucciso da Cadmo nascerà Echione, e quindi Penteo, e in drago si trasformeranno lo stesso Cadmo e la figlia Agave.

Il polimorfismo dionisiaco e la sua vicinanza al serpente, al toro ed altri animali spaventosi è un ulteriore richiamo alla sfera animale. Dioniso è invocato ad apparire come drago dalle sue menadi che agitano serpenti e se li intrecciano tra i capelli. L'affinità di Dioniso con l'animale, nel contesto delfico, potrebbe alludere ad una certa intercambiabilità del dio con la figura di Pitone ${ }^{44}$.

Nell'ambito di un'ideologia cosmologica, altrettanto forte è l'unione del serpente con la sfera acquatica. II serpente può rappresentare le divinità acquatiche primordiali. Così Oceano viene rappresentato con il corpo di un serpente 0 un serpente tra le mani.

Il potere nocivo delle creature serpentiformi è proprio delle entità mostruose della stirpe di $\operatorname{Pontos}^{45}$ e nei vasi canosini il rapporto sembra ulteriormente rafforzato dall'importanza del nesso Gorgo-serpenti su un contenitore come l'askòs funzionale a versare acqua ${ }^{46}$. L'introduzione di elementi acquatici ben veicola il concetto dello scorrere dell'acqua, come elemento primordiale, vivificatore.

L'esperienza pittorica italica fa emergere, con numerosi esempi, il valore paradigmatico dei serpenti, manifestazione di quanto sia insidioso l'Ade che attende il defunto. L'interpretazione del serpente come animale ctonio consente di comprendere il processo che nella pittura etrusca ne sottolinea il legame con le coppie alate di Vanth e di Charun. Nella tradizione iconografica etrusca, i demoni, armati di serpenti e fiaccole, sono altresì divinità del destino e forze selvagge proiettate sulla storia individuale del defunto. Come personificazioni del destino e come simboli dei fata, chiudono la scena, quali quinte teatrali 0 , in un'estrema evoluzione del messaggio simbolico legato alla posizione familiare del defunto, ne racchiudono il nome, sempre con gli orribili attributi dei serpenti tra le mani.

La raffigurazione pittorica nella breve sequenza sugli askòi canosini definisce, invero, una correlazione semantica tra soggetto e valore funzionale dei vasi, fondamentali nella composizione ceramica dei corredi dauni e nelle pratiche funerarie 4 .

II punto di discontinuità tra le credenze salvifiche legate agli apporti orfici e dionisiaci, che avevano contribuito ad esorcizzare nelle città italiote e negli ambienti ellenizzati le forze ostili del fato, e le credenze tardoellenistiche si manifesta nel mondo daunio con i caratteri intrinsecamente ambigui del nuovo repertorio figurativo.

La nascita di un lessico pittorico, in cui le forme di rappresentazione hanno una nuova cultura dell'immagine e uno specifico storico-religioso, coincide con le profonde trasformazioni delle comunità locali e con i nuovi orientamenti politico-sociali indotti dalla presenza di Roma.

\footnotetext{
${ }^{40}$ Auffarth 1991:359. Nei testi egizi è presente la visione circolare dell'universo vivente e il serpente viene definito il circondatore della terra.

${ }^{41}$ Auffarth 1991: 144.

42 Bodson 1978: 81.

43 Bodson 1990: 45-62.

44 Fontenrose 1959.

45 Baglioni 2010: 65-72.

${ }^{46}$ I morti domandano sia vino che acqua. Si veda in Omero il rito celebrato da Odisseo pressi i Cimmerii per evocare le ombre dei defunti: Hom.,Od.,XI, 24-28. In Platone è sostanziale la relazione di natura idrografica tra mondo umano ed infero: Plat.Phaed.112.

${ }^{47}$ La documentazione archeologica evidenzia, in molti contesti ipogeici ellenistici del territorio canosino, la collocazione degli askòi sulla soglia, con il collo rovesciato verso la terra. Si tratta di un rituale dal forte potere evocativo associato alle qualità intrinseche all'acqua, elemento puro ed agente purificatore, indispensabile alla sopravvivenza della comunità. L'entità materica della testimonianza archeologica illumina sulla dialettica purità/impurità legata all'acqua che scorre dal vaso come elemento fecondante e sulla ritualizzazione della soglia, come frontiera/cerniera tra mondi diversi.Si può ricordare come molti vasi a destinazione esclusivamente funeraria presenti nei corredi dauni siano privi di fondo, in un'evidente strategia di contatto con le cavità infere e di dialettica interrelazionale tra le libagioni e la casa di Hades.
} 
E' difficile dire se i serpenti con valore ctonio dei vasi canosini vadano visti come survival 48 di elementi organicamente appartenenti a presenze religiose interne alla cultura indigena o come esito di interazioni culturali. E' d'obbligo ricordare che l'attualizzazione dei motivi iconografici e le diverse forme stilistiche ravvisabili nell'artigianato daunio devono essere state favorite, nella prima metà del II sec. a.C., dalla intensificazione degli apporti culturali ed economici di tradizione italiota, italica e greco-orientale. Iconografie emblematiche afferenti alla sfera funeraria, rispondenti in parte ai parametri e agli interessi culturali della committenza gentilizia canosina, e in parte al sostrato sociale e culturale dei ceti intermedi e subalterni, possono quindi aver coerentemente arricchito il repertorio figurativo, in un processo di rielaborazione delle espressioni religiose locali e dei molteplici apporti di altre forme religiose.

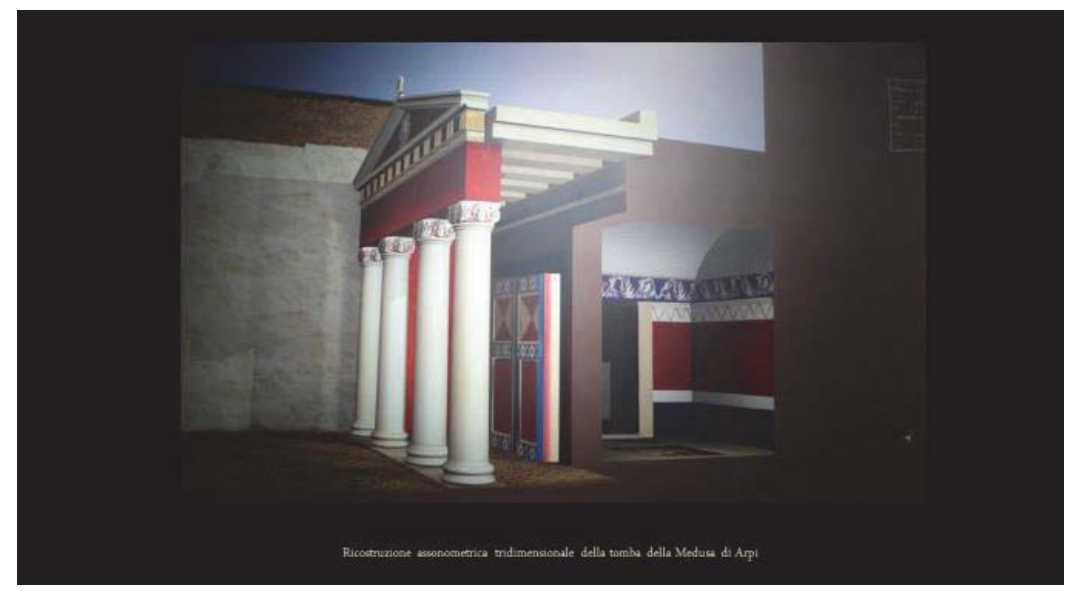

Fig.1. Arpi, tomba della Medusa

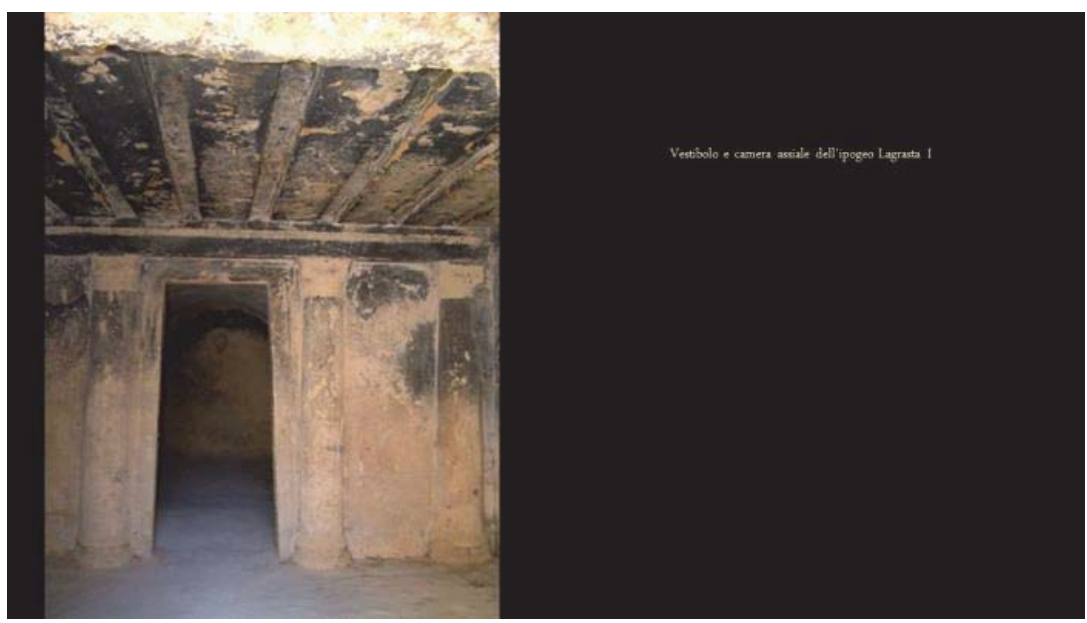

Fig.2. Canosa di Puglia, ipogeo Lagrasta I. Articolazione architettonica del vestibolo 


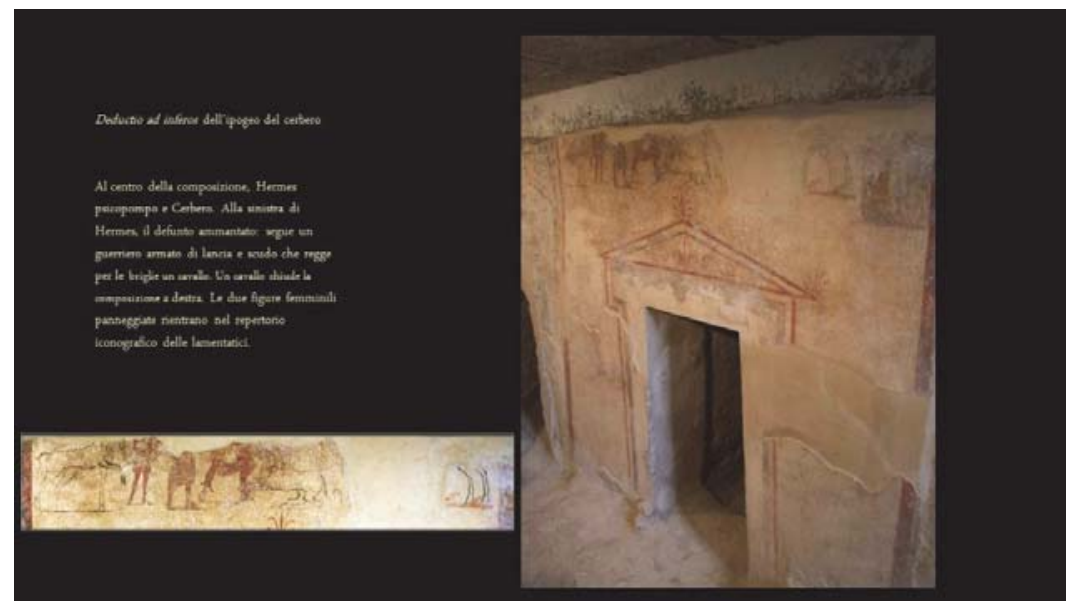

Fig. 3. Canosa di Puglia, ipogeo del Cerbero

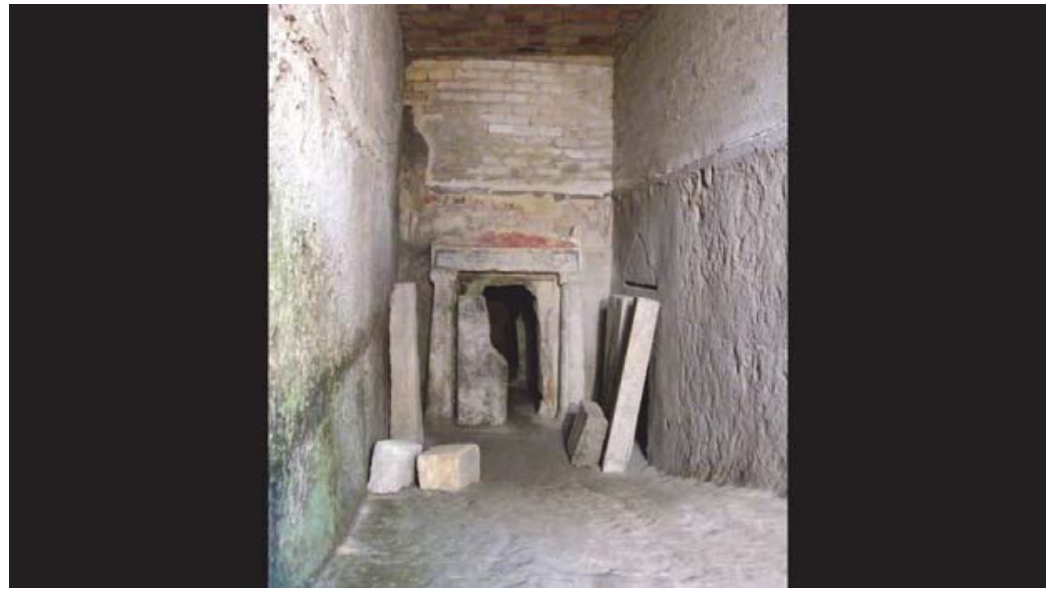

Fig. 4. Canosa di Puglia, ipogeo Scocchera B

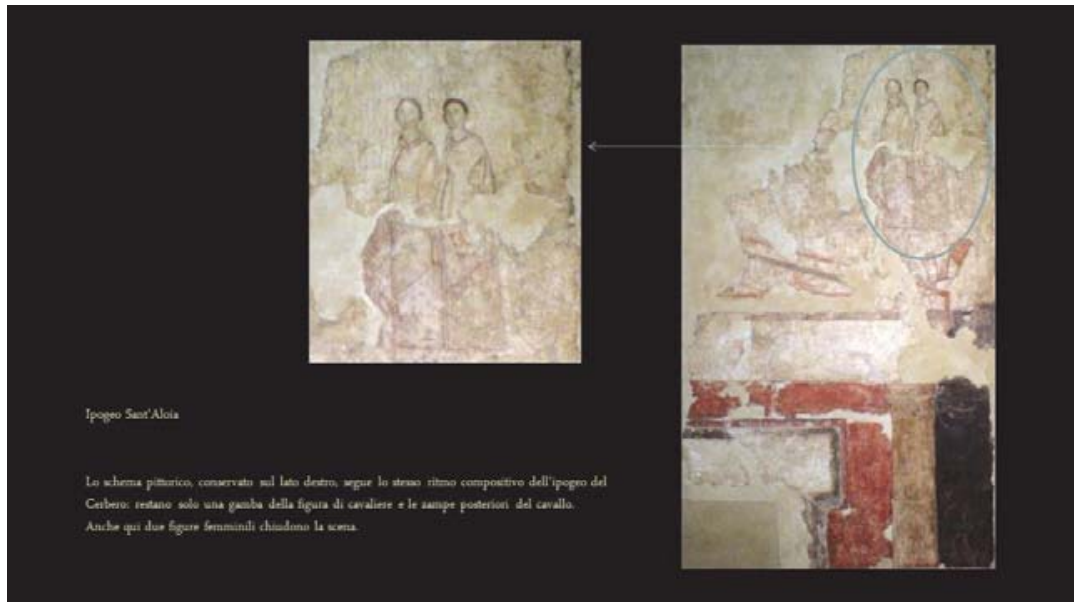

Fig. 5. Canosa di Puglia, ipogeo sant'Aloia 


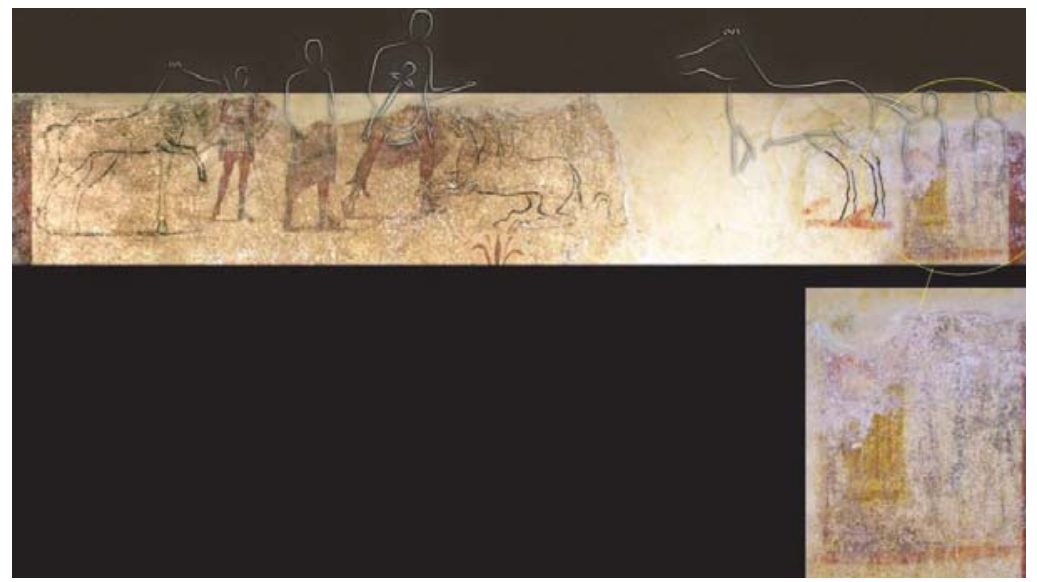

Fig. 6. Schema pittorico del fregio esterno dell'ipogeo del Cerbero
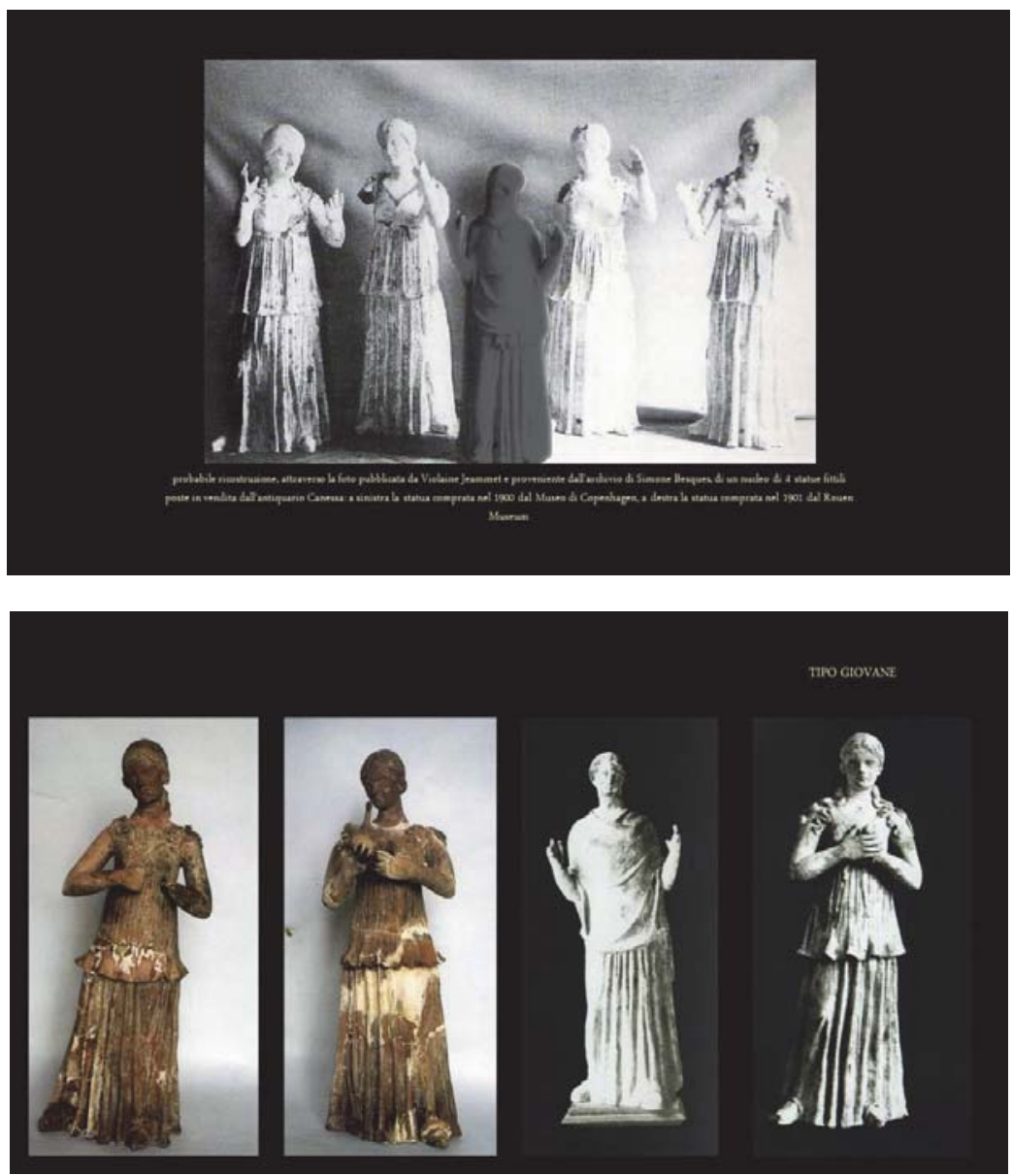

Figg. 7-8. Oranti fittili di produzione canosina 


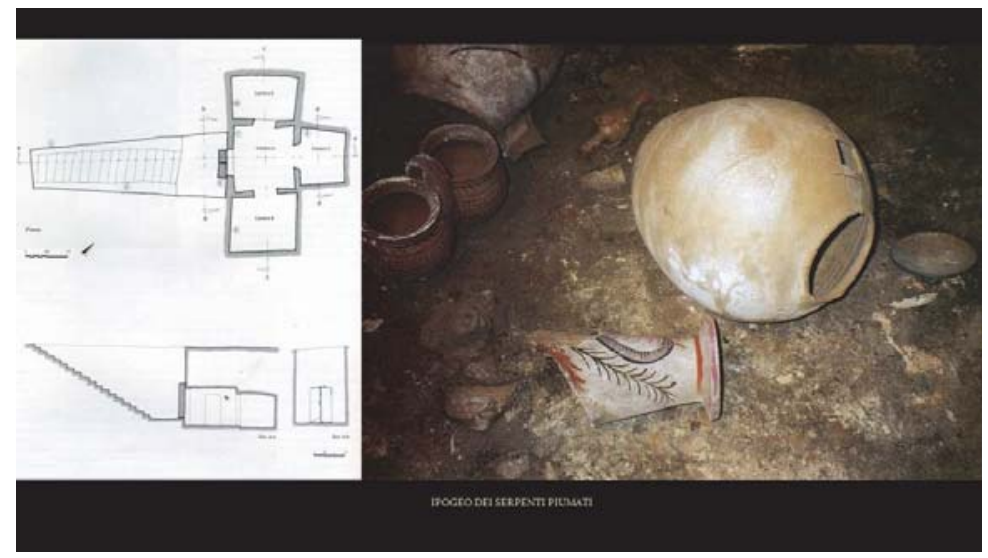

Fig. 9. Canosa di puglia. Corredo funerario dell'ipogeo dei serpenti piumati, con askos policromo
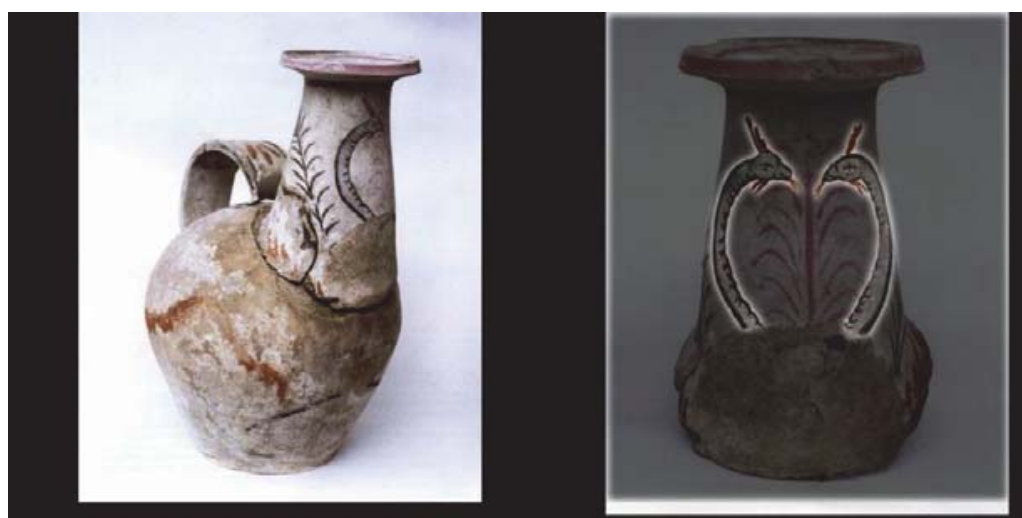

Fig. 10. Askos con serpenti in posizione araldica proveniente dall'ipogeo dei serpenti piumati

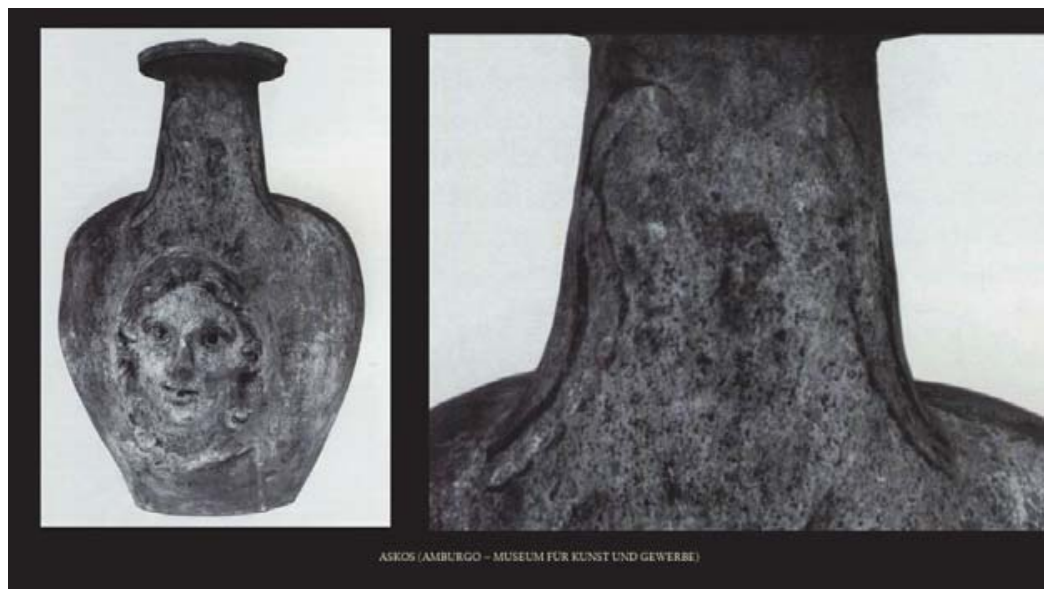

Fig. 11. Riproduzione dello stesso schema con coppia di serpenti sull'esemplare di Amburgo 